\title{
Iterative process for a strictly pseudo-contractive mapping in uniformly convex Banach spaces
}

\author{
Yu Zhou ${ }^{*}$, Haiyun Zhou ${ }^{1,2}$ and Peiyuan Wang ${ }^{1}$ \\ Dedicated to the 80th birthday of Professor Shih-Sen Chang
}

${ }^{*}$ Correspondence:

zhouyu871013@gmail.com

1 Department of Mathematics,

Shijiazhuang Mechanical

Engineering College, Shijiazhuang,

050003, China

Full list of author information is

available at the end of the article

\begin{abstract}
This paper is concerned with a new method to prove the weak convergence of a strictly pseudo-contractive mapping in a $p$-uniformly convex Banach space with more relaxed restrictions on the parameters. Our results extend and improve the corresponding earlier results.

MSC: Primary 41A65; 47H17; secondary 47J20

Keywords: strictly pseudo-contractive mapping; nonexpansive mapping; Mann iteration; uniformly convex Banach space
\end{abstract}

\section{Introduction and preliminaries}

In 1967, Browder and Petryshyn [1] gave the classical definition for strictly pseudocontractive mappings in Hilbert spaces for the first time.

Definition 1.1 Let $C$ be a nonempty closed convex subset of a real Hilbert space $H$. $T: C \rightarrow H$ is called a Browder-Petryshyn-type $k$-strictly pseudo-contractive mapping. Then there exists $k \in[0,1)$ such that for every $x, y \in C$

$$
\langle T x-T y, j(x-y)\rangle \leq\|x-y\|^{2}-k\|(I-T) x-(I-T) y\|^{2} .
$$

In 2010, Zhou [2] gave a new definition for $k$-strictly pseudo-contractive mappings in $q$-uniformly smooth Banach spaces.

Definition 1.2 Let $C$ be a nonempty closed convex subset of a $q$-uniformly smooth Banach space $X . T: C \rightarrow C$ is called a Zhou-type $k$-strictly pseudo-contractive mapping, if there exists $k \in[0,1)$ such that for every $x, y \in C$

$$
\left\langle T x-T y, j_{q}(x-y)\right\rangle \leq\|x-y\|^{q}-\frac{1-k}{2}\|(I-T) x-(I-T) y\|^{q} .
$$

In 2009, Hu and Wang [3] gave another definition for $k$-strictly pseudo-contractive mappings in $p$-uniformly convex Banach spaces.

@2014 Zhou et al.; licensee Springer. This is an Open Access article distributed under the terms of the Creative Commons Attribution License (http://creativecommons.org/licenses/by/2.0), which permits unrestricted use, distribution, and reproduction in any medium, provided the original work is properly cited. 
Definition 1.3 Let $C$ be a nonempty closed convex subset of a $p$-uniformly convex Banach space $X . T: C \rightarrow C$ is called a Hu-type $k$-strictly pseudo-contractive mapping, if there exists $k \in[0,1)$ such that for every $x, y \in C$

$$
\|T x-T y\|^{p} \leq\|x-y\|^{p}+k\|(I-T) x-(I-T) y\|^{p} .
$$

Remark 1.1 The mappings defined by (1.1) and (1.2) are pseudo-contractive mappings, but the mapping defined by (1.3) may not be pseudo-contractive in general Banach spaces.

Remark 1.2 If and only if $q=2$, the mappings defined by (1.1) and (1.2) are equivalent.

Remark 1.3 If $p=q=2$, the mappings defined by (1.1), (1.2), and (1.3) are equivalent in Hilbert space.

In 1979, Reich [4] established a weak convergence theorem via a Mann-type iterative process for nonexpansive mapping in a uniformly convex Banach space with Fréchet differentiable norm.

Theorem $\mathbf{R}$ Let $C$ be a closed convex subset of a uniformly convex Banach space $X$ with a Fréchet differentiable norm and $T: C \rightarrow C$ a nonexpansive mapping with $F(T) \neq \emptyset$. For any $x_{1} \in C$, the iterative sequence $\left\{x_{n}\right\}$ is defined by $x_{n+1}=\left(1-\alpha_{n}\right) x_{n}+\alpha_{n} T x_{n}$, where the real sequence $\left\{\alpha_{n}\right\} \subset[0,1]$ and $\sum_{n=1}^{\infty}\left(1-\alpha_{n}\right) \alpha_{n}=\infty$. Then the sequence $\left\{x_{n}\right\}$ converges weakly to a fixed point of $T$.

In 2007, Marino and Xu [5] improved Reich's [4] result and gave several weak convergence theorems via the normal Mann iterative algorithm for strictly pseudo-contractive mappings in Hilbert spaces. Further, they proposed an open problem: Do the main results of [5] still hold true in the framework of Banach spaces which are uniformly convex and have a Fréchet differentiable norm?

In 2009, Hu and Wang [3] considered above problem in a $p$-uniformly convex Banach space and established the following theorem.

Theorem $\mathbf{H}$ Let $C$ be a closed convex subset of a p-uniformly convex Banach space $X$ with a Fréchet differentiable norm and $T: C \rightarrow C$ be a $k$-strictly pseudo-contractive mapping in the light of (1.3) with coefficients $p, k<\min \left\{1,2^{-(p-2)} c_{p}\right\}$ and $F(T) \neq \emptyset$. For any $x_{1} \in C$ and $n>1$, the iterative sequence $\left\{x_{n}\right\}$ is defined by $x_{n+1}=\left(1-\alpha_{n}\right) x_{n}+\alpha_{n} T x_{n}$, where the real sequence $\left\{\alpha_{n}\right\} \subset[0,1]$ and $0<\varepsilon \leq \alpha_{n} \leq 1-\varepsilon<1-\frac{2^{p-2} k}{c_{p}}$. Then the sequence $\left\{x_{n}\right\}$ converges weakly to a fixed point of $T$.

Question Can one relax the restriction on the parameters $\alpha_{n}$ in Theorem $\mathrm{H}$ and simplify its proof?

The purpose of this paper is to solve the question mentioned above. To prove our results, we need the following lemmas.

Lemma 1.1 (see [3]) Let $C$ be a nonempty closed convex subset of a p-uniformly convex Banach space $X$ and $T: C \rightarrow C$ be a Hu-type strictly pseudo-contractive mapping in the 
light of (1.3). For $\alpha \in(0,1)$, define $T_{\alpha}: C \rightarrow C$ by $T_{\alpha}=(1-\alpha) x+\alpha T x$, for $x \in C$. If $\alpha \in$ $\left(0,1-\left(k 2^{p-2}\right) / c_{p}\right)$, then $T_{\alpha}$ is a nonexpansive mapping and $F\left(T_{\alpha}\right)=F(T)$.

Lemma 1.2 (see [3]) Let $C$ be a nonempty closed convex subset of a p-uniformly convex Banach space $X$ and $T: C \rightarrow C$ be a Hu-type strictly pseudo-contractive mapping in the light of (1.3). For $\mu \in(0,1), T_{\mu}: C \rightarrow C$ is defined by $T_{\mu}=(1-\mu) x+\mu T x$, for $x \in C$. Then the following inequality holds:

$$
\left\|T_{\mu} x-T_{\mu} y\right\|^{p} \leq\|x-y\|^{p}-\left(W_{p}(\mu) c_{p}-\mu \lambda\right)\|(I-T) x-(I-T) y\|^{p}, \quad \forall x, y \in C,
$$

where $W_{p}(\mu)=\mu^{p}(1-\mu)+\mu(1-\mu)^{p}$.

Lemma 1.3 (see [6]) Let $C$ be a nonempty closed convex subset of a p-uniformly convex Banach space $X$ and $T: C \rightarrow C$ be a nonexpansive mapping, then $I-T$ is demiclosed at zero.

Lemma 1.4 (see [7]) Let C be a nonempty closed convex subset of a p-uniformly convex Banach space $X$ which satisfies the Opial condition and $T: C \rightarrow C$ be a quasi-nonexpansive mapping with $F(T) \neq \emptyset$. If I-T is demiclosed at zero, then for any $x_{0} \in C$, the normal Mann iteration $\left\{x_{n}\right\}$ defined by

$$
x_{n+1}=\left(1-\alpha_{n}\right) x_{n}+\alpha_{n} T x_{n}, \quad \forall n \geq 0,
$$

converges weakly to a fixed point of $T$, where $\left\{\alpha_{n}\right\} \subset[0,1]$ and $\sum_{n=0}^{\infty} \min \left\{\alpha_{n},\left(1-\alpha_{n}\right)\right\}=\infty$.

Lemma 1.5 (see [7]) Let $C$ be a nonempty closed convex subset of a p-uniformly convex Banach space $X$ whose dual space $X^{*}$ satisfies Kadec-Klee property and $T: C \rightarrow C$ be a nonexpansive mapping with $F(T) \neq \emptyset$. Then, for any $x_{0} \in C$, the normal Mann iteration $\left\{x_{n}\right\}$ defined by

$$
x_{n+1}=\left(1-\alpha_{n}\right) x_{n}+\alpha_{n} T x_{n}, \quad \forall n \geq 0,
$$

converges weakly to a fixed point of $T$, where $\left\{\alpha_{n}\right\} \subset[0,1]$ and $\sum_{n=0}^{\infty} \min \left\{\alpha_{n},\left(1-\alpha_{n}\right)\right\}=\infty$.

Now we are in a position to state and prove the main results in this paper.

\section{Main results}

Theorem 2.1 Let $C$ be a nonempty closed convex subset of a p-uniformly convex Banach space $X$ with Fréchet differential norm. Let $T: C \rightarrow C$ be a Hu-type k-strictly pseudo-contractive mapping in the light of $(1.3)$ with coefficients $p, k<\min \left\{1,2^{-(p-2)} c_{n}\right\}$ and $F(T) \neq \emptyset$. Assume that a real sequence $\left\{\alpha_{n}\right\}$ in $[0,1]$ satisfies the conditions:

(i) $0 \leq \alpha_{n} \leq \alpha=1-\left(k 2^{p-2} / c_{p}\right), n \geq 0$;

(ii) $\sum_{n=0}^{\infty} \alpha_{n}\left[\left(1-\alpha_{n}\right) 2^{2-p} c_{p}-k\right]=\infty$.

For any $x_{0} \in C$, the normal Mann iterative sequence $\left\{x_{n}\right\}$ is defined by

$$
x_{n+1}=\left(1-\alpha_{n}\right) x_{n}+\alpha_{n} T x_{n}, \quad n \geq 0 .
$$

Then the sequence $\left\{x_{n}\right\}$ defined by (2.1) converges weakly to a fixed point of $T$. 
Proof Let $T_{\alpha}$ be given as in Lemma 1.1. Then $T_{\alpha}: C \rightarrow C$ is a nonexpansive mapping with $F\left(T_{\alpha}\right)=F(T)$. Set $\beta_{n}=\frac{\alpha-\alpha_{n}}{\alpha}$. Then (2.1) reduces to $x_{n+1}=\beta_{n} x_{n}+\left(1-\beta_{n}\right) T_{\alpha} x_{n}$.

We note that

$$
\begin{aligned}
\sum_{n=0}^{\infty} \beta_{n}\left(1-\beta_{n}\right) & =\frac{1}{\alpha^{2}} \sum_{n=0}^{\infty} \alpha_{n}\left(\alpha-\alpha_{n}\right) \\
& =\frac{1}{\alpha^{2}} \sum_{n=0}^{\infty} \alpha_{n}\left(1-\alpha_{n}-\frac{k 2^{p-2}}{c_{p}}\right) \\
& =\frac{2^{p-2}}{\alpha^{2} c_{p}} \sum_{n=0}^{\infty} \alpha_{n}\left[\left(1-\alpha_{n}\right) 2^{p-2} c_{p}-k\right] \\
& =\infty .
\end{aligned}
$$

By using Theorem R, we conclude that $\left\{x_{n}\right\}$ converges weakly to a fixed point of $T_{\alpha}$, and of $T$. The proof is complete.

Remark 2.2 Theorem 2.1 relaxes the iterative parameters in Theorem $\mathrm{H}$ and our proof method is also quite concise.

Theorem 2.3 Let $C$ be a nonempty closed convex subset of a p-uniformly convex Banach space $X$ which satisfies the Opial condition. Let $T: C \rightarrow C$ be a Hu-type $k$-strictly pseudo-contractive mapping in the light of $(1.3)$ with coefficients $p, k<\min \left\{1,2^{-(p-2)} c_{p}\right\}$ and $F(T) \neq \emptyset$. Assume that the real sequence $\left\{\alpha_{n}\right\}$ in $[0,1]$ satisfies the conditions:

(i) $0 \leq \alpha_{n} \leq \alpha=1-\left(k 2^{p-2} / c_{p}\right), n \geq 0$;

(ii) $\sum_{n=0}^{\infty} \alpha_{n}\left[\left(1-\alpha_{n}\right) 2^{2-p} c_{p}-k\right]=\infty$.

For any $x_{0} \in C$, the normal Mann iteration $\left\{x_{n}\right\}$ is defined by

$$
x_{n+1}=\left(1-\alpha_{n}\right) x_{n}+\alpha_{n} T x_{n}, \quad n \geq 0 .
$$

Then the sequence $\left\{x_{n}\right\}$ defined by (2.2) converges weakly to the fixed point of $T$.

Proof Let $T_{\alpha}$ be given as in Lemma 1.1. Then $T_{\alpha}: C \rightarrow C$ is a nonexpansive mapping with $F\left(T_{\alpha}\right)=F(T)$. Set $\beta_{n}=\frac{\alpha-\alpha_{n}}{\alpha}$. Then (2.2) reduces to $x_{n+1}=\beta_{n} x_{n}+\left(1-\beta_{n}\right) T_{\alpha} x_{n}$. As shown in Theorem 2.1, $\sum_{n=0}^{\infty} \beta_{n}\left(1-\beta_{n}\right)=\infty$. By Lemma 1.3, $I-T_{\alpha}$ is demiclosed at zero. By Lemma 1.4, we conclude that $\left\{x_{n}\right\}$ converges weakly to a fixed point of $T_{\alpha}$, and of $T$. The proof is complete.

Theorem 2.4 Let $C$ be a nonempty closed convex subset of a p-uniformly convex Banach space $X$ with the dual space $X^{*}$ satisfying the Kadec-Klee property. Let $T: C \rightarrow C$ be a Hu-type $k$-strictly pseudo-contractive mapping in the light of (1.3) with coefficients $p, k<$ $\min \left\{1,2^{-(p-2)} c_{p}\right\}$ and $F(T) \neq \emptyset$. Assume that the real sequence $\left\{\alpha_{n}\right\}$ in $[0,1]$ satisfies the conditions:

(i) $0 \leq \alpha_{n} \leq \alpha=1-\left(k 2^{p-2} / c_{p}\right), n \geq 0$;

(ii) $\sum_{n=0}^{\infty} \alpha_{n}\left[\left(1-\alpha_{n}\right) 2^{2-p} c_{p}-k\right]=\infty$. 
For any $x_{0} \in C$, the normal Mann iteration $\left\{x_{n}\right\}$ is defined by

$$
x_{n+1}=\left(1-\alpha_{n}\right) x_{n}+\alpha_{n} T x_{n}, \quad n \geq 0 \text {. }
$$

Then the sequence $\left\{x_{n}\right\}$ defined by (2.3) converges weakly to a fixed point of $T$.

Proof Let $T_{\alpha}$ be given as in Lemma 1.1. Then $T_{\alpha}: C \rightarrow C$ is a nonexpansive mapping with $F\left(T_{\alpha}\right)=F(T)$. Set $\beta_{n}=\frac{\alpha-\alpha_{n}}{\alpha}$. Then (2.3) reduces to $x_{n+1}=\beta_{n} x_{n}+\left(1-\beta_{n}\right) T_{\alpha} x_{n}$. As shown in Theorem 2.1, $\sum_{n=0}^{\infty} \beta_{n}\left(1-\beta_{n}\right)=\infty$. By using Lemma 1.5, $\left\{x_{n}\right\}$ defined by (2.3) converges weakly to a fixed point of $T_{\alpha}$, and of $T$. The proof is complete.

Competing interests

The authors declare that they have no competing interests.

Authors' contributions

All the authors contributed equally.

Author details

${ }^{1}$ Department of Mathematics, Shijiazhuang Mechanical Engineering College, Shijiazhuang, 050003, China. ${ }^{2}$ Department of Mathematics and Information, Hebei Normal University, Shijiazhuang, 050016, China.

\section{Acknowledgements}

This research was supported by the National Natural Science Foundation of China (11071053).

Received: 14 February 2014 Accepted: 7 August 2014 Published: 29 Sep 2014

\section{References}

1. Browder, FE, Petryshyn, WV: Contraction of fixed points of nonlinear mappings in Hilbert spaces. J. Math. Anal. Appl. 20, 82-90 (1967)

2. Zhou, HY: Convergence theorem for strict pseudo-contractions in uniformly smooth Banach spaces. Acta Math. Sin Engl. Ser. 26(4), 743-758 (2010)

3. Hu, LG, Wang, JP: Mann iteration of weak convergence theorem in Banach space. Acta Math. Sin. Engl. Ser. 25(2), 217-224 (2009)

4. Reich, S: Weak convergence theorem for nonexpansive mappings in Banach spaces. J. Math. Anal. Appl. 67, 274-276 (1979)

5. Marino, G, Xu, HK: Weak and strong convergence theorems for strict pseudo-contractions in Hilbert spaces. J. Math. Anal. Appl. 279, 336-349 (2003)

6. Browder, FE: Semicontractive and semiaccretive nonlinear mappings in Banach spaces. Bull. Am. Math. Soc. 74, 660-665 (1968)

7. Agarwal, RP, O'Regan, D, Sahu, DR: Fixed Point Theory for Lipschitzian-Type Mappings with Applications, pp. $299-302$. Springer, Berlin (2008)

10.1186/1029-242X-2014-377

Cite this article as: Zhou et al.: Iterative process for a strictly pseudo-contractive mapping in uniformly convex

Banach spaces. Journal of Inequalities and Applications 2014, 2014:377 